\title{
PRINCIPALLY ORDERED REGULAR SEMIGROUPS
}

\author{
by T. S. BLYTH and G. A. PINTO
}

(Received 30 May, 1989)

An ordered semigroup $S$ will be called principally ordered if, for every $x \in S$, there exists

$$
x^{*}=\max \{y \in S ; x y x \leq x\} .
$$

Here we shall be concerned with the case where $S$ is regular. We begin by listing some basic properties that arise from the above definition. As usual, we shall denote by $V(x)$ the set of inverses of $x \in S$.

$$
x^{\prime} \in V(x) \Rightarrow x^{\prime} \leq x^{*}
$$

This follows immediately from the fact that $x x^{\prime} x=x$.

$$
x=x x^{*} x \text {. }
$$

By (1), if $x^{\prime} \in V(x)$ then $x=x x^{\prime} x \leq x x^{*} x$, whence we have equality.

$$
x^{\circ}=x^{*} x x^{*} \text { is the greatest inverse of } x .
$$

In fact, by (2), we have $x^{*} x x^{*} \in V(x)$. If now $x^{\prime} \in V(x)$ then, by (1), $x^{\prime}=x^{\prime} x x^{\prime} \leq x^{*} x x^{*}$. Thus $x^{\circ}=x^{*} x x^{*}$ is the greatest inverse of $x$.

$$
x x^{\circ}=x x^{*} \text { is the greatest idempotent in } R_{x} .
$$

It is clear from (3) and (2) that $x x^{\circ}=x x^{*}$. Also, by (2), $x$ and $x x^{*}$ are $\mathscr{R}$-related. If now $e$ is an idempotent that is $\mathscr{R}$-related to $x$ then we have $e=x y$ and $x=e z$ for some $y, z \in S$. It follows that $x y x=e x=e z=x$ and so $y \leq x^{*}$ whence $e=x y \leq x x^{*}$.

$$
x^{\circ} x=x^{*} x \text { is the greatest idempotent in } L_{x} \text {. }
$$

This is similar to (4)

$$
x \leq x^{* *} \text { and } x \leq x^{\infty} \text {. }
$$

By (3) and (1), $x^{*} x x^{*}=x^{\circ} \leq x^{*}$ and so $x \leq x^{* *}$. The second inequality follows from (3) and the fact that $x$ is an inverse of $x^{\circ}$.

$$
x^{0 *}=x^{* *}
$$

This follows from the observation that

$$
\begin{aligned}
y \leq x^{\circ *} & \Leftrightarrow x^{\circ} y x^{\circ} \leq x^{\circ} \\
& \Leftrightarrow x^{*} x x^{*} y x^{*} x x^{*} \leq x^{*} x x^{*} \\
& \Leftrightarrow x x^{*} y x^{*} x \leq x \\
& \Leftrightarrow x^{*} y x^{*} \leq x^{*} \\
& \Leftrightarrow y \leq x^{* *} . \\
x^{*} & =x^{* * *}
\end{aligned}
$$

By (6) we have $x^{*} \leq x^{* * *}$. To obtain the reverse inequality, observe that

$$
x y x \leq x^{* *} \Rightarrow x^{*} x y x x^{*} \leq x^{*} \Rightarrow x y x \leq x
$$

Glasgow Math. J. 32 (1990) 349-364. 
and hence that

$$
x y x \leq x^{* *} \Leftrightarrow x y x \leq x .
$$

Now, by (6) and (2), we have

$$
x x^{* * *} x \leq x^{* *} x^{* * *} x^{* *}=x^{* *},
$$

and therefore, by the above observation,

$$
x x^{* * *} x \leq x
$$

and so $x^{* * *} \leq x^{*}$.

$$
x^{* 0}=x^{* *}
$$

We have

$$
\begin{array}{rlr}
x^{* 0} & =x^{* *} x^{*} x^{* *} & \\
& =x^{* *} x^{* * *} x^{* * *} & \text { by (8) } \\
& =x^{* *} & \text { by (2). } \\
& x^{\circ 0} \leq x^{* *} &
\end{array}
$$

This follows from the observation that

$$
\begin{aligned}
& x^{\infty}=x^{\circ *} x^{\circ} x^{\circ *} \quad \text { by (3) } \\
& =x^{* *} x^{0} x^{* *} \quad \text { by (7) } \\
& \leq x^{* *} x^{*} x^{* *} \quad \text { by (1) } \\
& =x^{* *} x^{* * *} x^{* *} \text { by }(8) \\
& =x^{* *} \quad \text { by (2). } \\
& x^{\circ}=x^{000}
\end{aligned}
$$

In fact,

$$
\begin{array}{rlrl}
x^{\circ}=x^{*} x x^{*} & =x^{*} x^{* *} x^{*} x x^{*} x^{* *} x^{*} \\
& =x^{*} x^{0 *} x^{\circ} x^{\circ *} x^{*} & \text { by (7) } \\
& =x^{*} x^{\circ \circ} x^{*} \\
& =x^{* * *} x^{\circ 0} x^{* * *} & \text { by ( } 8) \\
& =x^{00 *} x^{\circ 0} x^{\circ 0 *} & \text { by (7) } \\
& =x^{000} .
\end{array}
$$

EXAMple 1. A perfect Dubreil-Jacotin semigroup is characterised in [2] as an ordered regular semigroup $S$ in which

$(\alpha) x^{*}=\max \{y \in S ; x y x \leq x\}$ exists for every $x \in S$;

(B) $\xi=\max \left\{x \in S ; x^{2} \leq x\right\}$ exists;

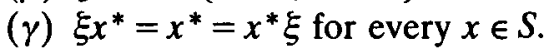

Every perfect Dubreil-Jacotin semigroup is therefore principally ordered. 
EXAmple 2. On the cartesian ordered set

$$
S=\left\{(x, y, z) \in \mathbb{Z}^{3} ; 0 \leq y \leq x\right\}
$$

define a multiplication by

$$
(x, y, z)(a, b, c)=(\sup \{x, a\}, y, z+c) .
$$

Then clearly $S$ is an ordered semigroup. It is regular since

$$
(x, y, z)(x, x,-z)(x, y, z)=(x, y, 0)(x, y, z)=(x, y, z) \text {. }
$$

The idempotents are the elements of the form $(x, y, 0)$, and as they form a subsemigroup $S$ is orthodox. Since

$$
\begin{aligned}
(x, y, z)(a, b, c)(x, y, z)=(\sup \{x, a\}, y, 2 z+c) & \leq(x, y, z) \\
& \Leftrightarrow a \leq x, c \leq-z \\
& \Leftrightarrow(a, b, c) \leq(x, x,-z),
\end{aligned}
$$

we see that $(x, y, z)^{*}=(x, x,-z)$ and so $S$ is principally ordered.

EXAMPLE 3 [The boot-lace]. Let $G$ be an ordered group and let $x \in G$ be such that $1<x$. Let $M=M(G ; I, \Lambda ; P)$ be the regular Rees matrix semigroup over $G$ with $I=\Lambda=\{1,2\}$ and sandwich matrix

$$
P=\left[\begin{array}{cc}
x^{-1} & 1 \\
1 & 1
\end{array}\right]
$$

With $\{1,2\}$ ordered by $1<2$, we can regard $P$ as an isotone mapping from the cartesian ordered set $\{1,2\} \times\{1,2\}$ to $G$. Recall that the multiplication in $M$ is given by

$$
(i, a, \lambda)(j, b, \mu)=\left(i, a p_{\lambda j} b, \mu\right) .
$$

The set of idempotents of $M$ is

$$
E=\{(1, x, 1),(1,1,2),(2,1,1),(2,1,2)\} .
$$

Let $\bar{E}=\bigcup_{n \geq 1} E^{n}$ be the subsemigroup generated by the idempotents. Then, with the convention that $x^{0}=1$, we have

$$
\bar{E}=\left\{\left(i, x^{n}, \lambda\right) ; i, \lambda \in\{1,2\}, n \in \mathbb{Z}\right\} .
$$

By a result of Fitz-Gerald [5], the set $V\left(E^{n}\right)$ of inverse of elements in $E^{n}$ is $E^{n+1}$. It follows that $\bar{E}$ is regular.

Consider the relation $\leq$ defined on $\bar{E}$ by

$$
\left(i, x^{n}, \lambda\right) \leq\left(i^{\prime}, x^{m}, \lambda^{\prime}\right) \Leftrightarrow\left\{\begin{array}{l}
n=m, i \leq i^{\prime}, \lambda \leq \lambda^{\prime} \\
\text { or } n+1=m, i \leq i^{\prime} \\
\text { or } n+1=m, \lambda \leq \lambda^{\prime} \\
\text { or } n+1<m .
\end{array}\right.
$$


It is readily verified that this is an order on $\bar{E}$ which gives the boot-lace Hasse diagram

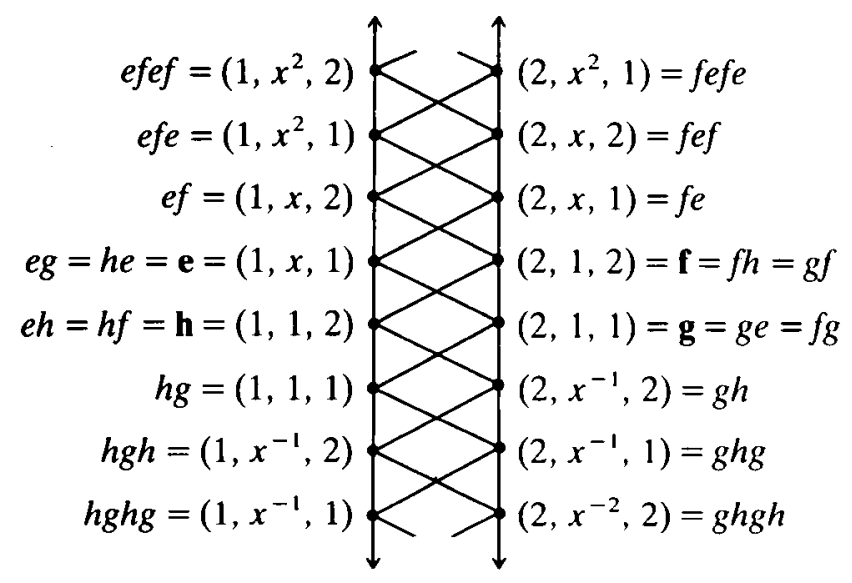

To see that $\bar{E}$ is thus an ordered semigroup, suppose that we have $\left(i, x^{n}, \lambda\right) \leq\left(i^{\prime}, x^{m}, \lambda^{\prime}\right)$ and compare

$$
\begin{gathered}
\left(i, x^{n}, \lambda\right)\left(j, x^{k}, \mu\right)=\left(i, x^{n} p_{\lambda j} x^{k}, \mu\right)=(i, \alpha, \mu), \\
\left(i^{\prime}, x^{m}, \lambda^{\prime}\right)\left(j, x^{k}, \mu\right)=\left(i^{\prime}, x^{m} p_{\lambda^{\prime} j} x^{k}, \mu\right)=\left(i^{\prime}, \beta, \mu\right) .
\end{gathered}
$$

If $n=m, i \leq i^{\prime}$ and $\lambda \leq \lambda^{\prime}$ then clearly $\alpha \leq \beta$ and $(i, \alpha, \mu) \leq\left(i^{\prime}, \beta, \mu\right)$. If $n+1=m$ and $i \leq i^{\prime}$ then $\alpha=\beta$ when $p_{\lambda j}=1$ and $p_{\lambda^{\prime} j}=x^{-1}$, in which case $(i, \alpha, \mu) \leq\left(i^{\prime}, \beta, \mu\right)$; otherwise, $\alpha=x^{a}<x^{b}=\beta$ with $a+1 \leq b$ in which case $(i, \alpha, \mu) \leq\left(i^{\prime}, \beta, \mu\right)$. If $n+1=m$ and $\lambda \leq \lambda^{\prime}$, or if $n+1<m$, then again we have $\alpha=x^{a}<x^{b}=\beta$ with $a+1 \leq b$ in which case $(i, \alpha, \mu) \leq\left(i^{\prime}, \beta, \mu\right)$. Thus we see that the multiplication in $\bar{E}$ is compatible on the right with the order; similarly, it is compatible on the left. have

The ordered regular semigroup $\bar{E}$ is principally ordered. In fact, for every $n \in \mathbb{Z}$ we

$$
\begin{aligned}
& \left(1, x^{n}, 1\right)^{*}=\left(1, x^{-n+2}, 1\right) \\
& \left(1, x^{n}, 2\right)^{*}=\left(2, x^{-n+1}, 1\right) ; \\
& \left(2, x^{n}, 1\right)^{*}=\left(1, x^{-n+1}, 2\right) ; \\
& \left(2, x^{n}, 2\right)^{*}=\left(2, x^{-n}, 2\right)
\end{aligned}
$$

To see the first of these, for example, observe that

$$
\left(1, x^{n}, 1\right)\left(1, x^{-n+2}, 1\right)\left(1, x^{n}, 1\right)=\left(1, x^{n}, 1\right)
$$

whereas for the element $\left(2, x^{-n+1}, 2\right)$, which is directly opposite $\left(1, x^{-n+2}, 1\right)$ in the Hasse diagram, we have

$$
\left(1, x^{n}, 1\right)\left(2, x^{-n+1}, 2\right)\left(1, x^{n}, 1\right)=\left(1, x^{n+1}, 1\right)>\left(1, x^{n}, 1\right) .
$$

It follows from this that

$$
\left(1, x^{n}, 1\right)^{*}=\left(1, x^{-n+2}, 1\right) \text {. }
$$

Similarly, we have the other formulae. Note that in this example we have $a=a^{* *}$ for every $a \in \bar{E}$. 
Simple calculations show that in both Examples 2 and 3 we have $x^{\circ}=x^{*}$ for every $x$. It is easy to construct further examples in which this identity does not hold.

Example 4. Consider the smallest non-orthodox naturally ordered regular semigroup with a greatest idempotent (see, for example, [3]). This is the semigroup $N_{5}$ described by the following Hasse diagram and Cayley table:

\begin{tabular}{ll|lllll}
$u$ & $u$ & $e$ & $f$ & $a$ & $b$ \\
\hline$a$ & $u$ & $u$ & $u$ & $f$ & $f$ & $b$ \\
$b$ & $e$ & $e$ & $e$ & $a$ & $a$ & $b$ \\
$f$ & $u$ & $b$ & $f$ & $b$ & $b$ \\
$a$ & $e$ & $b$ & $a$ & $b$ & $b$ \\
$b$ & $b$ & $b$ & $b$ & $b$ & $b$
\end{tabular}

It is readily seen that $x u x=x$ for every $x \in N_{5}$ and so $N_{5}$ is principally ordered with $x^{*}=u$ for every $x$. In fact, $N_{5}$ is perfect Dubreil-Jacotin. by

With $\bar{E}$ as in Example 3, define a multiplication on the cartesian ordered set $N_{5} \times \bar{E}$

$$
(p, x)(q, y)=(p q, x y) .
$$

Then $N_{5} \times \bar{E}$ is a principally ordered regular semigroup in which

$$
(p, x)^{*}=\left(u, x^{*}\right)
$$

In this semigroup, we have

$$
(p, x)^{\circ}=(p, x)^{*}(p, x)(p, x)^{*}=\left(u p u, x^{\circ}\right)=\left(u p u, x^{*}\right) .
$$

It follows that, for example,

$$
(b, x)^{\circ}=\left(b, x^{*}\right)<\left(u, x^{*}\right)=(b, x)^{*} .
$$

Note also that

$$
(p, x)^{* *}=(u, x), \quad(p, x)^{\circ o}=(u p u, x)
$$

and so in $N_{5} \times \bar{E}$ we have $x \neq x^{* *}$ and $x \neq x^{\circ 0}$ in general.

Bearing in mind Example 1 above, we recall from [2] that if $S$ is a perfect Dubreil-Jacotin semigroup then two particular features of $S$ are, on the one hand, that $S$ has a greatest idempotent and, on the other, that the assignment $x \mapsto x^{*}$ is antitone. In a general principally ordered regular semigroup, these two properties are independent.

To see this, we refer first to Example 3. Here we have a principally ordered regular semigroup in which the idempotents form the 4-element crown

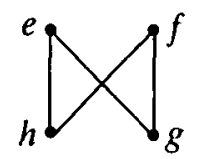

and so there is no greatest idempotent. But, as is readily verified, the assignment $x \mapsto x^{*}$ is antitone on $\vec{E}$. 
To obtain an example of a principally ordered regular semigroup that has a greatest idempotent and in which $x \mapsto x^{*}$ is not antitone, we consider a subsemigroup of that of Example 2.

EXAMPLE 5. Let $k$ be a fixed positive integer and consider the subset $T_{k}$ of the semigroup $S$ of Example 2 given by

$$
T_{k}=\left\{(x, y, z) \in \mathbb{Z}^{3} ; 0 \leq y \leq x \leq k\right\} .
$$

Then it is readily seen that $T_{k}$ is a principally ordered regular semigroup in which $(x, y, z)^{*}=(x, x,-z)$. Clearly, $T_{k}$ has a greatest idempotent, namely the element $(k, k, 0)$, and $x \mapsto x^{*}$ is not antitone.

Our main objective now is to show that for a principally ordered regular semigroup $S$ the conditions ( $a) S$ has a greatest idempotent, and $(b)$ the mapping $x \mapsto x^{*}$ is antitone, are necessary and sufficient for $S$ to be a perfect Dubreil-Jacotin semigroup. For this purpose, we first establish the following result.

THEOREM 1. Let $S$ be a principally ordered regular semigroup in which $x \mapsto x^{*}$ is antitone. Then $S$ is naturally ordered.

Proof. Let $\leq$ denote the natural order on the idempotents, so that

$$
e \leq f \Leftrightarrow e f=f e=e .
$$

Suppose that $e \leq f$. Then these equalities give $f e f=e$ and $e f e=e$. From the latter we obtain $f \leq e^{*}$ whence, by (6) and the fact that $x \mapsto x^{*}$ is antitone, we have $e \leq e^{* *} \leq f^{*}$. It follows that $e=f e f \leq f f^{*} f=f$. Thus the order on $S$ extends the natural order on the idempotents and so $S$ is naturally ordered.

Corollary. Let $S$ be a principally ordered regular semigroup in which $x \mapsto x^{*}$ is antitone. Then $S$ is locally inverse.

Proof. This follows immediately from [6, Proposition 1.4].

We recall now that if $E$ is the set of idempotents of $S$ and if $e, f \in E$ then the sandwich set $S(e, f)$ is defined by

$$
S(e, f)=\{g \in E ; g=g e=f g, e g f=e f\} .
$$

A characteristic property of locally inverse semigroups is that sandwich sets are singletons. We identify them in the present situation as follows.

THEOREM 2. Let $S$ be a principally ordered regular semigroup in which $x \mapsto x^{*}$ is antitone, and let $e, f \in E$. Then $S(e, f)=\left\{f(e f)^{\circ} e\right\}$.

Proof. Observe that if $g=f(e f)^{\circ} e$ then $g^{2}=g=g e=f g$ and $e g f=e f$.

Since the structure of naturally ordered regular semigroups with a greatest idempotent has been completely determined in [3], we shall focus our attention on principally ordered regular semigroups in which $x \mapsto x^{*}$ is antitone. Unless otherwise specified, $S$ will henceforth denote such a semigroup. For such a semigroup, we now list properties that will lead us to our goal.

$$
(\forall e \in E) e^{\infty} \in E \cap V(e)
$$


In fact, this was shown by Saito [7, Proposition 2.2] to hold in any naturally ordered regular semigroup in which greatest inverses exist, and therefore holds in the present situation by Theorem 1 and property (3) above.

$$
(\forall x, y \in S) \quad x y(x y)^{\circ} \leq x x^{\circ}, \quad(x y)^{\circ} x y \leq y^{\circ} y .
$$

From $y(x y)^{\circ} x y(x y)^{\circ}=y(x y)^{\circ}$ we deduce that

$$
x \leq\left[y(x y)^{\circ}\right]^{*},
$$

and so, by (6) and the fact that $x \mapsto x^{*}$ is antitone,

$$
y(x y)^{\circ} \leq\left[y(x y)^{\circ}\right]^{* *} \leq x^{*} .
$$

It follows that $x y(x y)^{\circ} \leq x x^{*}=x x^{\circ}$. Similarly, we can show that $(x y)^{\circ} x y \leq y^{\circ} y$.

$$
(\forall x, y \in S) \quad(x y)^{\circ}=\left(x^{\circ} x y\right)^{\circ} x^{\circ}=y^{\circ}\left(x y y^{\circ}\right)^{\circ} .
$$

Since, by (5), $x$ and $x^{\circ} x$ are $\mathscr{L}$-related, and since $\mathscr{L}$ is right compatible with multiplication, we have that $x y$ and $x^{\circ} x y$ are $\mathscr{L}$-related, whence

$$
\text { (a) }(x y)^{\circ} x y=\left(x^{\circ} x y\right)^{\circ} x{ }^{\circ} x y .
$$

It follows that

$$
x y \cdot\left(x^{\circ} x y\right)^{\circ} x^{\circ} \cdot x y=x y(x y)^{\circ} x y=x y
$$

and so

$$
\left(x^{\circ} x y\right)^{\circ} x^{\circ} \leq(x y)^{*}
$$

Using this, we see that

$$
\begin{array}{rlr}
x y(x y)^{\circ} & =x y(x y)^{\circ} x y(x y)^{\circ} \\
& =x y\left(x^{\circ} x y\right)^{\circ} x^{\circ} x y(x y)^{\circ} & \text { by }(a) \\
& \leq x y\left(x^{\circ} x y\right)^{\circ} x^{\circ} x x^{\circ} & \text { by }(13) \\
& =x y\left(x^{\circ} x y\right)^{\circ} x^{\circ} \\
& \leq x y(x y)^{*} \\
& =x y(x y)^{\circ},
\end{array}
$$

whence we have

$$
\text { (b) } x y(x y)^{\circ}=x y\left(x^{\circ} x y\right)^{\circ} x^{\circ} \text {. }
$$

It now follows that

$$
\begin{aligned}
& (x y)^{\circ}=(x y)^{\circ} x y(x y)^{\circ} \\
& =\left(x^{\circ} x y\right)^{\circ} x^{\circ} x y(x y)^{\circ} \quad \text { by }(a) \\
& =\left(x^{\circ} x y\right)^{\circ} x^{\circ} x y\left(x^{\circ} x y\right)^{\circ} x^{\circ} \text { by }(b) \\
& =\left(x^{\circ} x y\right)^{\circ} x^{\circ} \text {. }
\end{aligned}
$$

Similarly, we can show that $(x y)^{\circ}=y^{\circ}\left(x y y^{\circ}\right)^{\circ}$.

$$
(\forall x \in S) \quad\left(x x^{\circ}\right)^{\circ}=x^{\circ} x^{\circ}, \quad\left(x^{\circ} x\right)^{\circ}=x^{\circ} x^{\circ} .
$$

Take $y=x^{\circ}$ in the first equality of (14), and $x=y^{\circ}$ in the second. 
Remark. Note that (13) and (14) were established by Saito [7] in the case of a naturally ordered regular semigroup with greatest inverses and in which Green's relations $\mathscr{R}$ and $\mathscr{L}$ are regular, in the sense that

$$
x \leq y \Rightarrow x x^{\circ} \leq y y^{\circ} \text { and } x \leq y \Rightarrow x^{\circ} x \leq y^{\circ} y .
$$

Later, we shall see the significance of the regularity of Green's relations $\mathscr{R}$ and $\mathscr{L}$ in the present context of a principally ordered regular semigroup in which the mapping $x \mapsto x^{*}$ is antitone.

$$
\text { If } e \in E \text { and } f^{2} \leq f \text { then } e \leq f \Rightarrow e=e f e .
$$

This follows from the observation that

$$
\begin{aligned}
& e \leq f \Rightarrow f e f e f \leq f^{5} \leq f \\
& \Rightarrow e f e \leq f^{*} \leq e^{*} \\
& \Rightarrow e f e \leq e e^{*} e=e \\
& \Rightarrow e \leq f \leq e^{*} \\
& \Rightarrow e \leq e f e \leq e e^{*} e=e \\
& \Rightarrow e=e f e . \\
&(\forall e \in E) \quad e^{\circ} \in E \Leftrightarrow e^{*} \in E .
\end{aligned}
$$

For every $e \in E$ we have

$$
\begin{aligned}
e^{\circ} \in E & \Leftrightarrow e^{\circ} e^{\circ}=e^{\circ} \\
& \Leftrightarrow e^{*} e e^{*} \cdot e^{*} e e^{*}=e^{*} e e^{*} \\
& \Leftrightarrow e e^{*} e^{*} e=e .
\end{aligned}
$$

Thus $e^{\circ} \in E$ implies that $e^{*} e^{*} \leq e^{*}$. But $e^{\circ} \in E$ also implies, by (5), (7) and (1), that

$$
e^{\circ} \leq e^{\circ} e^{\circ}=e^{o *} e^{\circ}=e^{* *} e^{\circ} \leq e^{* *} e^{*}
$$

whence, by (16),

$$
e^{* *} e^{*} \leq e^{0 *}=e^{* *} \leq e^{*},
$$

the last inequality following from the fact that $e \leq e^{*}$. It follows that

$$
e^{*}=e^{*} e^{* *} e^{*} \leq e^{*} e^{*}
$$

Thus $e^{\circ} \in E$ implies that $e^{*} \in E$. Conversely, if $e^{*} \in E$ then $e e^{*} e^{*} e=e e^{*} e=e$ and, from the above, we deduce that $e^{\circ} \in E$.

For every $x \in S$ we now consider the elements

$$
\begin{gathered}
\alpha_{x}=x^{\circ \circ} x^{\circ} \in E, \quad \beta_{x}=x^{\circ} x^{\circ} \in E . \\
(\forall x \in S) \quad \alpha_{x}=\alpha_{x}^{\circ} \leq \alpha_{x}^{*} \in E, \quad \beta_{x}=\beta_{x}^{\circ} \leq \beta_{x}^{*} \in E .
\end{gathered}
$$

Using (15) and (11), we observe that

$$
\alpha_{x} \leq \alpha_{x} \alpha_{x}^{\circ}=x^{\infty} x^{\circ}\left(x^{\infty} x^{\circ}\right)^{\circ}=x^{\infty} x^{\circ} x^{\infty} x^{\circ}=x^{\infty} x^{\circ}=\alpha_{x},
$$


and so $\alpha_{x}=\alpha_{x} \alpha_{x}^{\circ}$. Similarly, we have $\alpha_{x}=\alpha_{x}^{\circ} \alpha_{x}$. It follows that

$$
\alpha_{x}=\alpha_{x} \alpha_{x}^{\circ}=\alpha_{x}^{\circ} \alpha_{x} \alpha_{x}^{\circ}=\alpha_{x}^{\circ},
$$

and so $\alpha_{x}^{\circ} \in E$. It now follows by (17) that $\alpha_{x}^{*} \in E$.

$$
\begin{aligned}
& \alpha_{x}^{*}=\left(x x^{*}\right)^{*} \text { is the greatest idempotent above } x x^{*}, \text { and } \\
& \beta_{x}^{*}=\left(x^{*} x\right)^{*} \text { is the greatest idempotent above } x^{*} x .
\end{aligned}
$$

Clearly, $x x^{*}=x x^{\circ} \leq x^{\circ} x^{\circ}=\alpha_{x} \leq \alpha_{x}^{*}$ where, by (18), $\alpha_{x}^{*} \in E$. Suppose now that $g \in E$ is such that $x x^{*} \leq g$. Then we have, using (7) and (15),

$$
g^{* *} \geq\left(x x^{*}\right)^{* *}=\left(x x^{\circ}\right)^{\circ *}=\left(x^{\infty} x^{\circ}\right)^{*}=\alpha_{x}^{*} \geq \alpha_{x},
$$

and so $g \leq g^{*} \leq \alpha_{x}^{*}$. Thus $\alpha_{x}^{*}$ is the greatest idempotent above $x x^{*}$.

The maximality of the idempotent $\alpha_{x}^{*}$ implies that

$$
\alpha_{x}^{*}=\alpha_{x}^{*} \alpha_{x}^{* *}=\alpha_{x}^{* *} \alpha_{x}^{*}
$$

and so

$$
\alpha_{x}^{* *}=\alpha_{x}^{* *} \alpha_{x}^{*} \alpha_{x}^{* *}=\alpha_{x}^{*} \alpha_{x}^{* *}=\alpha_{x}^{*}
$$

It follows by (7), (8), and (15) that

$$
\alpha_{x}^{*}=\alpha_{x}^{* *}=\left(x^{\circ 0} x^{\circ}\right)^{* *}=\left(x x^{\circ}\right)^{\circ * *}=\left(x x^{\circ}\right)^{* * *}=\left(x x^{\circ}\right)^{*}=\left(x x^{*}\right)^{*}
$$

The statement concerning $\beta_{x}^{*}$ is proved similarly.

$$
(\forall e, f \in E) \quad S(e, f)^{*}=\max \left\{x \in S ; e x f \leq(e f)^{* *}\right\} .
$$

By Theorem 2, $S(e, f)=\left\{f(e f)^{\circ} e\right\}=\{g\}$, say. Then

$$
\begin{aligned}
x \leq g^{*} & \Rightarrow g x g \leq g \\
& \Rightarrow f(e f)^{\circ} e \cdot x \cdot f(e f)^{\circ} e \leq f(e f)^{\circ} e \\
& \Rightarrow e f(e f)^{*} \cdot e x f .(e f)^{*} e f \leq e f \\
& \Rightarrow(e f)^{*} \operatorname{exf}(e f)^{*} \leq(e f)^{*} \\
& \Rightarrow e x f \leq(e f)^{* *} .
\end{aligned}
$$

Conversely, suppose that $e x f \leq(e f)^{* *}=(e f)^{\circ *}$. Then

$$
\begin{aligned}
g x g & =f(e f)^{\circ} e \cdot x \cdot f(e f)^{\circ} e \\
& \leq f(e f)^{\circ}(e f)^{\circ *}(e f)^{\circ} e \\
& =f(e f)^{\circ} e \\
& =g
\end{aligned}
$$

and hence $x \leq g^{*}$.

Using the above results, we can now establish the following:

THEOREM 3. Let $S$ be a principally ordered regular semigroup in which $x \mapsto x^{*}$ is antitotone. Then the following statements are equivalent:

(i) $(\forall e \in E) \quad e^{*}=e^{* *}$;

(ii) $(\forall e \in E) \quad e^{*} \in E$;

(iii) $S$ has a greatest idempotent;

(iv) $S$ is a perfect Dubreil-Jacotin semigroup. 
Proof. (i) $\Rightarrow\left(\right.$ ii): If $e^{*}=e^{* *}$ then $e^{*} e^{*} e^{*} \leq e^{*}$. But, by (16), $e \leq e^{*} e^{* *}$ gives $e^{*} e^{* *} \leq e^{*}$, so we have $e^{*}=e^{*} e^{* *} e^{*} \leq e^{*} e^{*}$ and so

$$
e^{*} \leq e^{*} e^{*} \leq e^{*} e^{*} e^{*} \leq e^{*}
$$

whence $e^{*}=e^{*} e^{*}$.

(ii) $\Rightarrow$ (iii): If (ii) holds then it is clear from (16) that, for every $e \in E$, $e^{*}$ is the greatest idempotent above $e$. Suppose now that $e, f \in E$. Then, by (ii), we have $e^{*}, f^{*} \in E$. Observe now from (20) and (ii) that $e^{*} \leq S\left(e^{*}, f^{*}\right)^{*} \in E$ and $f^{*} \leq S\left(e^{*}, f^{*}\right)^{*} \in E$. The maximiality of $e^{*}, f^{*}$ now gives $e^{*}=S\left(e^{*}, f^{*}\right)^{*}=f^{*}$. Since this holds for all $e, f \in E$, it follows that $S$ has a greatest idempotent.

(iii) $\Rightarrow($ iv $)$ : Suppose now that $\xi$ is the greatest idempotent in $S$. Then if $x \in S$ is such that $x^{2} \leq x$ we have

$$
x x x^{*} x=x x \leq x,
$$

which gives $x x^{*} \leq x^{*}$ and hence

$$
x=x x^{*} x \leq x^{*} x \leq \xi
$$

Thus $\xi=\max \left\{x \in S ; x^{2} \leq x\right\}$.

Now since $\xi$ is the greatest idempotent of $S$ it follows from (19) that $\xi=\alpha_{x}^{*}$ for every $x \in S$. Thus we have

$$
x^{*} \xi=x^{*} \alpha_{x}^{*}=x^{*}\left(x x^{*}\right)^{*}
$$

and hence

$$
x x^{*} \xi x x^{*}=x x^{*}\left(x x^{*}\right)^{*} x x^{*}=x x^{*}
$$

and consequently

$$
x x^{*} \xi x=x .
$$

This gives on the one hand $x^{*} \xi \leq x^{*}$. But, on the other hand, we have $x^{*}=x^{*} x^{* *} x^{*} \leq$ $x^{*} \xi$. Hence we see that $x^{*} \xi=x^{*}$ for all $x \in S$, and similarly $\xi x^{*}=x^{*}$.

Thus we see that conditions $(\beta),(\gamma)$ of $[2$, Theorem 1] hold and therefore $S$ is a perfect Dubreil-Jacotin semigroup.

(iv) $\Rightarrow(i)$ : This is immediate from the fact that in a perfect Dubreil-Jacotin semigroup we have $x^{*}=\xi: x$ and, for every idempotent $e, e^{*}=e^{* *}$ (see $\left.[2,(8),(9)]\right)$.

THEOREM 4. Let $S$ be a principally ordered regular semigroup in which $x \mapsto x^{*}$ is antitone. Then $S^{\circ}=\left\{x^{\circ} ; x \in S\right\}$ is a subsemigroup of $S$ with the same properties.

Proof. Let $a, b \in S^{\circ}$ so that, by (11), $a=a^{\circ \circ}$ and $b=b^{\circ}$. By (13), we have $a b(a b)^{\circ} \leq a a^{\circ}$ and so, by (19),

$$
\alpha_{a}^{*}=\left(a a^{\circ}\right)^{*} \leq\left[a b(a b)^{\circ}\right]^{*}=\left[a b(a b)^{*}\right]^{*}=\alpha_{a b}^{*} .
$$

By the maximality of $\alpha_{a}^{*}$, it follows that

$$
\left(a a^{\circ}\right)^{*}=\left[a b(a b)^{\circ}\right]^{*}
$$


Consequently, we have

$$
\begin{aligned}
(a b)^{\circ \circ}(a b)^{\circ} a b & =\left[a b(a b)^{\circ}\right]^{\circ} a b \quad \text { by }(15) \\
& =\left[a b(a b)^{\circ}\right]^{\circ} a b(a b)^{\circ} a b \\
& =\left[a b(a b)^{\circ}\right]^{*} a b(a b)^{\circ} a b \\
& =\left(a a^{\circ}\right)^{*} a b \\
& =\left(a a^{\circ}\right)^{*} a a^{\circ} a b \\
& =\left(a a^{\circ}\right)^{\circ} a a^{\circ} a b \\
& =\left(a a^{\circ}\right)^{\circ} a b \\
& =a^{\circ \circ} a^{\circ} a b \quad \text { by }(15) \\
& =a b \text { since } a=a^{\circ \circ},
\end{aligned}
$$

and similarly $a b=a b(a b)^{\circ}(a b)^{\infty}$. It follows that

$$
a b=(a b)^{\circ \circ}(a b)^{\circ} a b(a b)^{\circ}(a b)^{\circ \circ}=(a b)^{\circ \circ},
$$

and so $a b \in S^{\circ}$. Thus $S^{\circ}$ is a subsemigroup. It is principally ordered with $x \mapsto x^{*}$ antitone since for every $x^{\circ} \in S^{\circ}$ we have, by (7) and (9), $x^{\circ *}=x^{* *}=x^{* \circ} \in S^{\circ}$.

THEOREM 5. Let $S$ be a principally ordered regular semigroup in which $x \mapsto x^{*}$ is antitone. Then the following statements are equivalent:

(1) $S^{\circ}$ is orthodox;

(2) $S^{\circ}$ is inverse;

(3) $S^{\circ}$ has a greatest idempotent;

(4) $S$ is perfect Dubreil-Jacotin.

Proof. (1) $\Rightarrow(4)$ : Suppose that $S^{\circ}$ is orthodox and let $e \in E$. Then, observing that $e^{\circ} e^{\infty \circ} \in E \cap S^{\circ}$ and $e^{\infty \circ} e^{\circ} \in E \cap S^{\circ}$, we have, using (12),

$$
e^{\circ}=e^{\circ} e^{\infty} e^{\circ}=e^{0} e^{\infty} \cdot e^{\infty} e^{o} \in E .
$$

It follows by (17) that $e^{*} \in E$ and then by Theorem 3 that $S$ is a perfect Dubreil-Jacotin semigroup.

$(4) \Rightarrow(2)$ : In a perfect Dubreil-Jacotin semigroup we have $x^{*}=\xi: x$ and $x^{\circ}=$ $(\xi: x) x(\xi: x)$. If $e \in E$ then $\xi: e=\xi$ and so $e^{\circ}=\xi e \xi$. Since, as shown in [2], we have $e=e e^{*} e=e \xi e$, it follows that $e^{\circ} \in E \cap S^{\circ}$ and that $e^{\circ}=e^{\circ}$. Now if $f$ is an idempotent in $S^{\circ}$ then clearly $f=f^{\circ}=f^{\circ}$, and so every idempotent $f \in S^{\circ}$ is of the form $g^{\circ}$ for some idempotent $g\left(=f^{\circ}\right) \in S$. Now since $\xi S \xi$ is inverse we have $e^{\circ} f^{\circ}=f^{\circ} e^{\circ}$ for all $e, f \in E$. Consequently, the idempotents of $S^{\circ}$ commute and so $S^{\circ}$ is inverse.

$(2) \Rightarrow(1)$ : this is clear.

(3) $\Rightarrow$ (4): For every $e \in E$ we have $e \leq e^{\circ \circ} \in E$, by (12). Thus, if $S^{\circ}$ has a greatest idempotent then so must $S$, whence $S$ is perfect Dubreil-Jacotin by Theorem 3 .

(4) $\Rightarrow$ (3): If $S$ is perfect Dubreil-Jacotin then, by [2], $\xi=\xi^{\circ} \in S^{\circ}$ and is the greatest idempotent.

EXAMPLE 6. In the semigroup $N_{5} \times \bar{E}$ of Example 4 we have $(p, x)^{\circ}=\left(u p u, x^{*}\right)$. It follows that

$$
\left(N_{5} \times \bar{E}\right)^{\circ}=\left\{\left(b, x^{*}\right),\left(u, x^{*}\right) ; x \in \bar{E}\right\}=\{b, u\} \times \bar{E},
$$

which is not orthodox. 
For every idempotent $e \in S$ we know that $e \leq e^{\circ} \leq e^{*}$. We now investigate some consequences of equality occurring.

An idempotent $e$ is maximal if and only if $e=e^{*}$.

If $e$ is a maximal idempotent then clearly $e=e e^{\circ}=e^{\circ} e$ and so $e^{\circ}=e^{\circ} e e^{\circ}=e e^{\circ}=e \in E$. It follows by (17) that $e^{*} \in E$ and hence that $e=e^{*}$ by the maximality of $e$ in $E$. Conversely, if $e=e^{*}$ then $e=e e^{*}$ and so

$$
e=e^{*}=\left(e e^{*}\right)^{*}=\alpha_{e}^{*}
$$

whence, by (19), $e$ is a maximal idempotent.

$$
\begin{aligned}
& \text { If } e, f \in E \text { are such that } e=e^{\circ} \text { and } f=f^{\circ} \text { then } \\
& \qquad(e f)^{\circ}=S(e, f) \in E .
\end{aligned}
$$

Two applications of (14) give

$$
(e f)^{\circ}=f^{\circ}\left(e^{\circ} e f f^{\circ}\right)^{\circ} e^{\circ}=f(e f)^{\circ} e=S(e, f) \in E .
$$

If $e, f$ are maximal idempotents then we have the Hasse diagram

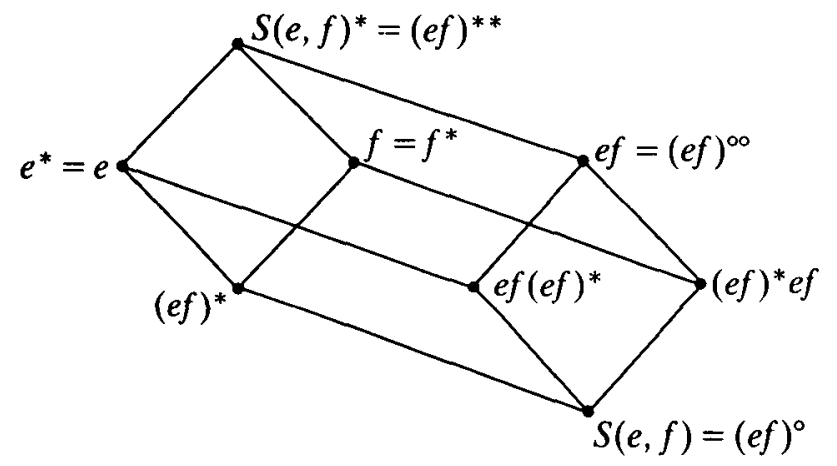

By (21), we have $e=e^{\circ}=e^{*}$ and $f=f^{\circ}=f^{*}$. Now since the idempotent $S(e, f)$ absorbs $e$ on the right we have $e . S(e, f) \in E$, and indeed $e . S(e, f) \leq e$ whence $e . S(e, f) \leq e$ by Theorem 1. Thus $e . S(e, f) . e \leq e$ and so $S(e, f) \leq e^{*}=e$. Similarly, we have $S(e, f) \leq f$. As $S(e, f) \in E$, we deduce that $S(e, f) \leq e f$. By Theorem 4 , ef $=e^{\circ} f^{\circ} \in S^{\circ}$ and so $e f=(e f)^{\circ}$. Now, by (22) we have $S(e, f)=(e f)^{\circ} \leq e$, which gives $e=e^{*} \leq S(e, f)^{*}=$ $(e f)^{\circ *}=(e f)^{* *}$ by $(7)$, and then $(e f)^{*} \leq e^{*}=e$. Similarly, $(e f)^{*} \leq f$. Consider now the idempotent $(e f)^{*} e f$. We have

$$
\text { (a) }(e f)^{*} e f \leq e . e f=e f \text {, }
$$

and, by (13),

$$
\text { (b) }(e f)^{*} e f \leq f^{*} f=f \text {. }
$$

Similarly, $e f(e f)^{*} \leq e f$ and $e f(e f)^{*} \leq e$. Finally, since $(e f)^{\circ}=S(e, f) \in E$ we have

$$
(e f)^{\circ} \leq(e f)^{\circ}(e f)^{\circ \circ}=(e f)^{\circ} e f=(e f)^{*} e f
$$

and likewise $(e f)^{\circ} \leq e f(e f)^{*}$. The diagram now follows by (1) and (10). 
THEOREM 6. Let $S$ be a principally ordered regular semigroup in which $x \mapsto x^{*}$ is antitone. If $S$ is not a perfect Dubreil-Jacotin semigroup then $S$ necessarily contains a crown of idempotents of the form

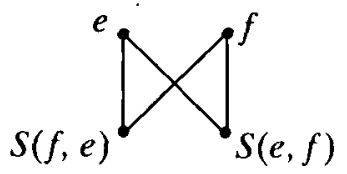

in which e, $f$ are maximal.

Moreover, if these are the only idempotents in $S$ then they generate the boot-lace semigroup.

Proof. If $S$ is not perfect Dubreil-Jacotin then, by (19) and Theorem $3, S$ must contain at least two maximal idempotents $e, f$. Now for these idempotents we must have $S(e, f)$ incomparable to $S(f, e)$. For example, if we had

$$
S(e, f)=(e f)^{\circ} \leq(f e)^{\circ}=S(f, e)
$$

then it would follow by (13) that

$$
f e(e f)^{\circ} \leq f e(f e)^{\circ} \leq f f^{\circ}=f
$$

and consequently

$$
f e f=f e S(e, f) f=f e(e f)^{\circ} f \leq f,
$$

which gives the contradiction $e \leq f^{*}=f$. It now follows by (23) and the corresponding diagram involving the product $f e$ that $S$ contains a 4-element crown of idempotents as described.

Suppose now that these are the only idempotents in $S$. Writing $S(e, f)=(e f)^{\circ}=g$ and $S(f, e)=(f e)^{\circ}=h$, we clearly have $g=f g=g e$ and $h=h f=e h$. Now the idempotent $(e f)^{\circ}$ ef cannot be equal to $g=(e f)^{\circ}$, for if this were so we would have

$$
e f=e f(e f)^{\circ} e f=e f g=e f(e f)^{\circ} \in E
$$

whence $e f \leq(e f)^{\circ}=g \leq e$ which gives $e f e \leq e$ and the contradiction $f \leq e^{*}=e$. It follows that we must have $(e f)^{\circ} e f=f$ and therefore

$$
g f=(e f)^{\circ} f=(e f)^{\circ} e f=f .
$$

Likewise, $f e(f e)^{\circ}=f$ and

$$
f h=f(f e)^{\circ}=f e(f e)^{\circ}=f .
$$

In a similar way we can show that $e g=h e=e$.

Observe now that $f e \geq h e=e, f e \geq f h=f$ and $e f \geq e g=e, e f \geq g f=f$. Moreover, $e, f$, $e f$, $f e$ are distinct since otherwise $e f \in E$ and this implies, by the maximality of $e$ and $f$, the contradiction $e=f$. Next, observe that $e f e \geq e f, f e$ and $f e f \geq e f, f e$ with $e f, f e$, efe, fef distinct (since otherwise we have that $e f e \in E$ whence the contradiction $e=f$ ). Continuing this argument, and doing so similarly with the minimal idempotents $g$ and $h$, we see that the semigroup generated by the idempotents is the boot-lace semigroup as described in Example 3. 
Corollary. Let PA denote the class of principally ordered regular semigroups in which $x \mapsto x^{*}$ is antitone and let PDJ denote the class of perfect Dubreil-Jacotin semigroups. Then the boot-lace is the semigroup in PA\PDJ with the least number of idempotents.

Further properties concerning the subsemigroup $\bar{E}$ generated by the set $E$ of idempotents are the following. Note that if $x \in \bar{E}$ then $x^{\circ} \in \bar{E}$; this follows by [5].

THEOREM 7. Let $S$ be a principally ordered regular semigroup in which $x \mapsto x^{*}$ is antitone. Then $S$ is a perfect Dubreil-Jacotin semigroup if and only if $\bar{E}$ is periodic.

Proof. $\Rightarrow$ : If $S$ is perfect Dubreil-Jacotin then for every $e \in E$ we have $\xi: e=\xi$. It follows by [1, Theorem 25.5] that $\xi: x=\xi$ for every $x \in \bar{E}$. Thus, for every $x \in \bar{E}$, we have

$$
x=x(\xi: x) x=x \xi x,
$$

and so $\xi$ is a medial idempotent in the sense of [4], by Theorem 1.1 of which it follows that $\bar{E}$ is periodic.

$\Leftarrow$ : Let $\bar{E}$ be periodic and let $e, f$ be maximal idempotents of $S$. Observe that by (a) of (23) we have

$$
e f=e f(e f)^{n} e f \leq(e f)^{2} .
$$

As $\bar{E}$ is periodic, it follows that for some positive integer $n$ we have

$$
e f \leq(e f)^{2} \leq \ldots \leq(e f)^{n} \in E .
$$

Consequently, by $(a),(b)$ of $(23)$, by $(16)$, and by the maximality of $f$, we see that

$$
\text { ef } \leq(e f)^{n} \leq\left[(e f)^{*} e f\right]^{*}=f^{*}=f,
$$

and similarly $e f \leq e$. By $(a)$ again, we then have $(e f)^{*} e f \leq e$ whence

$$
e=e^{*} \leq\left[(e f)^{*} e f\right]^{*}=f^{*}=f \text {. }
$$

The maximality of $e$ now gives $e=f$. Since this holds for all maximal idempotents $e, f$ it follows that $S$ has a greatest idempotent and so, by Theorem 3 , is perfect DubreilJacotin.

COROLlary. If $\bar{E}$ is finite then $S$ is perfect Dubreil-Jacotin.

Proof. If $\bar{E}$ is finite then it is necessarily periodic.

Finally, we turn our attention to the regularity of Green's relations $\mathscr{L}$ and $\mathscr{R}$. Here we have the following characterisation.

THEOREM 8. Let $S$ be a principally ordered regular semigroup in which $x \mapsto x^{*}$ is antitone. Then the following statements are equivalent:

(1) $\mathscr{L}$ is regular on $\bar{E}$;

(2) $\mathscr{R}$ is regular on $\bar{E}$;

(3) $S$ is a perfect Dubreil-Jacotin semigroup.

Proof. (1) $\Rightarrow(3)$ : Suppose that $\mathscr{L}$ is regular on $\bar{E}$. If $e, f \in E$ are such that $e \leq f$ then $e^{\circ} e \leq f^{\circ} f$ and so

$$
\beta_{f}^{*}=\left(f^{\circ} f\right)^{*} \leq\left(e^{\circ} e\right)^{*}=\beta_{e}^{*}
$$


where $f \leq f^{\circ} f \leq \beta_{f}^{*} \leq \beta_{e}^{*}$. Consequently, $\beta_{e}^{*}$ is the greatest idempotent above $e$. It follows that $\alpha_{e}^{*} \leq \beta_{e}^{*}$ whence, by the maximality of $\alpha_{e}^{*}$, we have

$$
\alpha_{e}^{*}=\beta_{e}^{*}=\gamma_{e} \text {, say. }
$$

Now $e \leq \gamma_{e}$ gives, by (16), $e=e \gamma_{e} e$ and so $\left(e \gamma_{e}\right)^{2}=e \gamma_{e} R e$. But $e e^{\circ}$ is the greatest idempotent in the $\mathscr{R}$-class of $e$, and $e e^{\circ} \leq e \gamma_{e}$. Hence $e e^{\circ}=e \gamma_{e}$, whence $e^{\circ}=e^{\circ} e \gamma_{e}$. Similarly, we have $e^{\circ} e=\gamma_{e} e$ and so $e^{\circ}=\gamma_{e} e e^{\circ}$. It now follows that

$$
e^{\circ} e^{\circ}=e^{\circ} e \gamma_{e} \cdot \gamma_{e} e e^{\circ}=e^{\circ} \cdot e \gamma_{e} e \cdot e^{\circ}=e^{\circ} e e^{\circ}=e^{\circ}
$$

and so $e^{\circ} \in E$. By (17) we deduce that $e^{*} \in E$ and then, by Theorem 3, that $S$ is perfect Dubreil-Jacotin.

(3) $\Rightarrow(1)$ : If $S$ is perfect Dubreil-Jacotin then, denoting the elements of $\bar{E}$ by $\bar{x}$, we have

$$
\bar{e} \leq \bar{f} \Rightarrow \bar{e}^{\circ} \bar{e}=\bar{e}^{*} \bar{e}=\xi \bar{e} \leq \xi \bar{f}=\bar{f}^{\circ} \bar{f}
$$

so that $\mathscr{L}$ is regular on $\bar{E}$.

The proof of $(1) \Leftrightarrow(2)$ is similar.

We remark here that although, in a perfect Dubreil-Jacotin semigroup $S$, Green's relations $\mathscr{R}$ and $\mathscr{L}$ are regular on the subsemigroup generated by the idempotents, they are not in general regular on $S$. This is illustrated in the following example.

Example 7. Consider a fixed integer $k>1$. For every $n \in \mathbb{Z}$ let $n_{k}$ denote the largest multiple of $k$ that is less than or equal to $n$, so that we have

$$
n_{k}=t k \leq n<(t+1) k \text {. }
$$

It is readily seen that $\mathbb{Z}$, under the usual order and the law of composition described by $(m, n) \mapsto m+n_{k}$, is a principally ordered regular semigroup in which $n^{*}=-n_{k}+k-1$. The mapping $x \mapsto x^{*}$ is then antitone. Since the idempotents are $0,1, \ldots, k-1$ it follows that this semigroup is perfect Dubreil-Jacotin.

Consider now the cartesian ordered semigroup $N_{5} \times \mathbb{Z}$ where $N_{5}$ is as in Example 4 . This is clearly non-orthodox and perfect Dubreil-Jacotin. By Theorem $8, \mathscr{R}$ and $\mathscr{L}$ are regular on the subsemigroup generated by the idempotents. Now for every $(x, n) \in N_{\mathrm{S}} \times \mathbb{Z}$ we have

$$
\begin{aligned}
& (x, n)(x, n)^{*}=(x, n)\left(u,-n_{k}+k-1\right)=\left(x u, n-n_{k}\right) ; \\
& (x, n)^{*}(x, n)=\left(u,-n_{k}+k-1\right)(x, n)=(u x, k-1) .
\end{aligned}
$$

It is clear from these equalities that $\mathscr{L}$ is regular on $N_{5} \times \mathbb{Z}$, whereas $\mathscr{R}$ is not.

\section{REFERENCES}

1. T. S. Blyth and M. F. Janowitz, Residuation Theory, Pergamon Press, 1972.

2. T. S. Blyth, Perfect Dubreil-Jacotin semigroups. Proc. Roy. Soc. Edinburgh, 78A, 1977, 101-104.

3. T. S. Blyth and R. McFadden, Naturally ordered regular semigroups with a greatest idempotent. Proc. Roy. Soc. Edinburgh, 91A, 1981, 107-122.

4. T. S. Blyth and R. McFadden, On the construction of a class of regular semigroups. Journal of Algebra, 81, 1983, 1-22. 
5. D. G. Fitz-Gerald, On inverses of products of idempotents in regular semigroups. $J$. Austral. Math. Soc., 13, 1972, 335-337.

6. D. B. McAlister, Regular Rees matrix semigroups and Dubreil-Jacotin semigroups. J. Austral. Math. Soc., 31, 1981, 325-336.

7. Tatsuhiko Saito, Naturally ordered regular semigroups with maximum inverses. Proc. Edinburgh Math. Soc., 32, 1989, 33-39.

Department of Mathematical Sciences, UNIVERSITY OF ST ANDREWS, ST ANDREWS KY16 9SS,

FIFE, SCOTLAND.

Departamento de Matematica, UNIVERSIDADE NOVA DE LISBOA, 2825 MONTE DA CAPARICA, Portugal. 